\title{
BRIEF ABSTRACTS OF ADDITIONAL POSTER PAPERS PRESENTED AT IAU COLLOQUIUM 113
}

\section{AGES OF LUMINOUS BLUE VARIABLES FROM THEIR NEBULAR AND}

STELLAR ENVIRONMENT, M.-C. Lortet.

A study of the spatial distribution of different kinds of young objects in the Magellanic Clouds shows that $S$ Dor-like stars (in a broad sense) are not the very youngest ones. A few specific ideas are developed in a longer article in these proceedings. Maps will be produced and discussed elsewhere.

\section{CURRENT OBSERVATIONS OF THE HUBBLE-SANDAGE VARIABLES IN M 31}

AND M 33, R.M. Humphreys, K. Davidson, O. Stahl, B. Wolf, and F.-J. Zickgraf. Recent moderate- and high-resolution spectra of $\mathrm{H}-\mathrm{S}$ variables covering $4000--9000 \AA$ have been obtained, giving estimates of mass-loss rates and electron densities in the ejected shells. From [NII] and [Fe III] emission in the red spectrum of AE And, we estimate the total mass of the [N II]-emitting gas to be at least $0.006 M_{\odot}$. This is very likely the mass ejected during its last outburst, and is additional evidence that the mean mass-loss rate during the total LBV phase is greater than the typical $10^{-4}$ to $10^{-5} M_{\odot} / \mathrm{yr}$.

QUANTITATIVE SPECTROSCOPY OF B-SUPERGIANTS IN THE SMC, LMC, AND GALAXY, H.G. Groth, R.P, Kudritzki, K. Butler, D. Husfeld, S. Becker, F. Eber, \& E. Fitzpatrick.

High-resolution, high-S/N spectra of nine la supergiants (B0 to B2) taken with the ESO $3.6 \mathrm{~m}$ telescope and CASPEC are analyzed by NLTE methods. All objects are found to have helium-enriched atmospheres, which points to enhanced mixing of $\mathrm{CN}$ burned material during the early evolution of massive stars. This is confirmed by the NLTE abundance determinations for two of the objects, which both show clear nitrogen enhancement.

\section{VARIABILITY OF THE O8Ib(f)p STAR HD 167971 IN THE INFRARED, C. Chavarria-K., C. Leitherer, L. Terranegra, and E. de Lara.}

The triple-star system HD 167971 was observed during nine photometric nights in the summers of 1987 and 1988 . The mean photometric values are: $K=5.18 \pm 0.02$, $\mathrm{J}-\mathrm{H}=0.28 \pm 0.01, \mathrm{H}-\mathrm{K}=0.14 \pm 0.01, \mathrm{H}-\mathrm{L}=0.25 \pm 0.04, \mathrm{~K}-\mathrm{M}=0.41 \pm 0.06$; the errors reflect the observed variability. Variability in $K$ follows the same pattern as in $\mathrm{V}$, except that the amplitude in $\mathrm{K}$ is smaller by a factor of two. The flux excesses in the $\mathrm{L}$ and $\mathrm{M}$ bandpasses are larger than those found by Leitherer et al. (1987, Astron. Astrophys. 185, 121), suggesting that the mass-loss rate is variable on timescales of one year or less.

WINDS IN CLOSE BINARY STARS: UW CMa (O7 Iaf + OB), M.F. Corcoran \& S.R. Heap.

UV spectra from the IUE and X-ray images from the Einstein Observatory reveal that: (1) The primary is the source of the observed photospheric and wind spectrum; (2) The secondary periodically occults portions of the wind; (3) The mass-loss rate has maxima at the primary's tidal bulges; (4) Material from the primary's outer tidal bulge expands more slowly than does the bulk wind; (5) $L_{X}=2 \times 10^{32} \mathrm{erg} / \mathrm{s}$, independent of orbital phase. 
THE INFLUENCE OF CLOSE BINARY EVOLUTION ON THE DISTRIBUTION

OF MASSIVE STARS IN THE H-R DIAGRAM, D. Vanbeveren.

A statistical study is carried out on the distribution of different kinds of massive star classes in the H-R diagram, using recent calculations of massive single-star and massive close-binary evolution. The influence of the mass tranfer process during the critical Roche lobe overflow phase of a primary component on the relative frequencies of different classes is outlined. It is shown that without an exact knowledge of how mass transfer takes place, the meaning of an initial mass function determined by classical methods is unclear, while a direct comparison of the observed and theoretically predicted blue/red star ratios is meaningless.

\section{PULSATING B STARS IN THE MAGELLANIC CLOUDS: FIRST RESULTS FOR}

THE LMC, C. Sterken \& M. Jerzykiewicz.

We have undertaken a search program for detecting $\beta$ Cephei stars in the LMC. Three program stars are found to exhibit short-term brightness variations. There is one promising $\beta$ Cep candidate: star 257 in the catalog of Brunet et al. (1975) shows a sinusoidal light curve with an amplitude (half-range) of $0.0070 \mathrm{mag}$ and a period of about 5 hours.

RAPID VARIABILITY IN O STAR WINDS, H. Henrichs, L. Kaper, \& R. Zwarthoed. Rapid variability is seen in the Si IV P Cygni profiles in high-resolution IUE spectra of three O stars: $\lambda$ Cep, O6 If; $68 \mathrm{Cyg}, 07.5 \mathrm{III}$; and $19 \mathrm{Cep}, 09.5 \mathrm{Ib}$. The variability occurs in the form of discrete absorption features, which march from low to high velocity within a day to a week (depending on the star), indicative of rapid changes in the stellar wind structure. The origin of this variability is not clear, but we confirm earlier suggestions that the beginning of a new 'episode' might be correlated with the rotation period of the star. There is growing evidence that the above behavior is in fact representative of all $O$ stars.

\section{TRANSIENT FEATURES IN THE He I P CYGNI PROFILES OF TWO EXTREME Of STARS, A.W. Fullerton, D.R. Gies, \& C.T. Bolton.}

Variable absorption features have been detected in the absorption trough of the He I $\lambda 5876$ P Cygni profiles of two luminous Of stars, HD 151804 and HD 152408. These features propagate from red to blue over several days. They resemble the discrete opacity enhancements observed in UV wind lines of $O$ stars, and are likely caused by the same mechanism.

THE WINDS OF CHEMICALLY EVOLVED O STARS, I.D. Howarth \& R.K. Prinja. Results of a differential analysis to investigate the surface chemistry of ONC, supergiant, and of stars are presented, based on a quantitative ultraviolet survey of the winds of 203 galactic O stars (Howarth \& Prinja 1989, Astrophys.J.Suppl.). The enhanced surface nitrogen abundance in ON stars is confirmed, while nitrogen appears to be depleted (by a factor of 5 ) in the OC stars. O supergiants do not exhibit nonsolar atmospheric abundances, but of stars appear to have mild $(<2 \times)$ surface nitrogen enhancements and may reveal $\mathrm{CNO}$-processed material. 
ON THE USE OF H $\alpha$ AND He II $\lambda 4686$ AS MASS-LOSS INDICATORS IN

LUMINOUS BLUE STARS, A. Gabler, R. Gabler, \& R.P. Kudritzki.

Our 'unified' model atmosphere code, which avoids the artificial division between photosphere and wind, is used to calculate correct theoretical $\mathrm{H} \alpha$ and $\mathrm{He}$ II $\lambda 4686$ profiles by varying the wind parameters for a given model ( $T_{\mathrm{eff}}, g, R$ fixed). The results can be used directly to derive mass-loss rates from observed profiles or equivalent widths.

\section{RATES OF MASS LOSS IN THE UPPER H-R DIAGRAM, H. Nieuwenhuijzen \& C. de Jager.}

We have used 258 observed values for the rate of stellar mass loss in the upper part of the H-R diagram, in conjunction with masses derived from evolutionary calculations, to search for simple parametrizations of $\log \dot{M}$. While a previously derived 20 -term expression gave an overall O-C standard deviation of $\sigma=0.49$ in $\log \dot{M}$, we now find $\sigma$ only $20 \%$ larger for the expression: $\log \dot{M}=8.78 \times 10^{-15} L^{1.24} M^{0.18} R^{0.81}$, where $L, M$, and $R$ are in solar units and $\log \dot{M}$ is in solar masses per year.

\section{UNIFIED NLTE MODEL ATMOSPHERES INCLUDING STELLAR WINDS AND} SPHERICAL EXTENSION, R. Gabler, A. Gabler, A. Pauldrach, J. Puls, \& R.P. Kudritzki.

Self-consistent model atmospheres are presented which use only $g$ and $L$ as free parameters. The model atmospheres -- calculated for hot Of-supergiants and O-giants -produce energy distributions and typical 'wind lines' (He II $\lambda 4686$, He II $\lambda 1640, P \alpha$, $B \alpha$ ) together with normal photospheric lines, as observed in stars of this type.

\section{RADIATION DRIVEN WINDS OF HOT LUMINOUS STARS -- THE LUMINOSITY} DEPENDENCE OF STELLAR WIND LINES, A. Pauldrach, R.P. Kudritzki, \&J. Puls. A grid of wind models along evolutionary tracks of massive $O$ stars is calculated using the improved radiation-driven wind theory, in order to investigate changes of spectral morphology during the course of stellar evolution. The results show that the improved stellar wind models can describe the significantly different observed behavior of the profiles of the Si IV line with respect to those of CIV and NV as a function of spectral type and luminosity class. Agreement with observations is also found for the computed mass-loss rates and the ratios of calculated terminal wind velocities and escape velocities. For the wind efficiencies, a strong dependence on mass was found, together with a pronounced decrease from main sequence stars to supergiants and a dramatic increase toward LBV's.

\section{ON THE STABILITY OF RADIAL PULSATIONS OF MASSIVE STARS,}

$J$. Klapp \& M. Corona.

We investigate the radial stability of massive stars using the classical Ledoux quasiadiabatic approximation, the Castor quasi-adiabatic approximation, and a fully nonadiabatic calculation. We find that the three methods of calculation give similar results provided that a small region in the outer layers of the star is neglected for the classical approximation. The critical mass for stability of massive stars is found to be in agreement with previous work. We explain the reason for the discrepancy between this and previous work by one of the authors. 


\section{LUMINOSITY AND RADIUS RELATIONS FOR WOLF-RAYET STARS,} N. Langer.

On the basis of a comprehensive set of numerical models for $\mathrm{W}-\mathrm{R}$ stars, we demonstrate that all observable properties of WNE/WC/WO-type stars depend solely on their mass and surface chemical composition. This enables one to derive very accurate relations for luminosities and radii as a function of these two parameters for various W-R subtypes ( $c f . \mathrm{N}$. Langer, Astron. Astrophys., in press).

\section{WC STARS: HOT STARS WITH COOL WINDS AND A STRATIFIED IONIZATION STRUCTURE, D.J. Hillier.}

Detailed modeling of WC4-6 stars confirms a C/He abundance of $>0.1$ (by number). The importance of dielectronic recombination for C III $\lambda 2296$ can explain the discrepancies between the abundances derived from UV lines and those based on analyses of optical recombination lines. Dielectronic recombination is also very important for the formation of the strong optical C III lines. Because of the high $\mathrm{C} / \mathrm{He}$ abundance, line cooling is very efficient. Typically we find $T_{\mathrm{e}}=9000 \mathrm{~K}$ at $N_{\mathrm{e}}=10^{9} \mathrm{~cm}^{-3}$ and $T_{\mathrm{e}}=$ $20000 \mathrm{~K}$ at $N_{\mathrm{e}}=10^{11} \mathrm{~cm}^{-3}$. The ionization state of the gas varies with radius.

\section{SPECTRAL ANALYSIS OF WN STARS IN THE LMC, W. Schmutz \& P.S. Conti.} Besides the sophisticated modeling of a particular W-R stellar spectrum (e.g. Hamann et al. 1988, Astron. Astrophys. 194, 190), a relatively simple way has been introduced of inferring W-R atmospheric parameters using model grids. From a few observed quantities, the stellar parameters of the W-R stars can be determined. Schmutz et al. (1988, Astron. Astrophys., in press) have published the stellar parameters for 30 galactic W-R stars. Here, the same procedure is applied to WN stars in the LMC. Based on the preliminary analysis presented in this poster, no systematic differences are found between galactic and LMC W-R stars.

\section{THE EVOLUTIONARY STATUS OF HD 62910 (WN6-C4),}

A.J. Willis \& D.J. Stickland.

A detailed analysis of high-resolution UV (IUE) and optical spectra of HD 62910 confirms its WN6-C4 composite classification, and shows that both its WN and WC features are formed in the same stellar wind. Abundances derived from a non-LTE Sobolev analysis indicate a zero $\mathrm{H}$-content and $\mathrm{He} / \mathrm{C} / \mathrm{N}$ ratios intermediate between those of WNE and WC stars. We conclude that HD 62910 is on its way from the WN6 to the WCE stage.

\section{TWELVE NIGHTS OF LINE PROFILE VARIATIONS OF HD 191765,}

S.R. McCandliss \& B. Bohannan.

HD 191765 (WN6) was observed for twelve nights over essentially the entire visible wavelength region to search for correlated line profile variations. No convincing periodic variations were found in either the line flux or line centroid measures. Periodograms of the flux variations have more power on time scales of a few hours, a time scale characteristic of the wind flow. Line profile variations of the Pickering series of HeII indicate that lines formed farther out in the wind suffer more variation than those formed deeper. Our results suggest that no single physical mechanism is responsible for the line profile variations. 
CCD OBSERVATIONS OF EMISSION LINE PROFILE VARIATIONS IN

WOLF-RAYET STARS, G. Koenigsberger \& P. Massey.

We report preliminary results of the observations of HD 164270 (WC9), HD 177230 (WN8), HD 192163 (WN6), and HD 193077 (WN6) in the wavelength range 4600-$4720 \AA$, at a resolution of $0.3 \AA$. Variations on a day-to-day timescale are detected in the four systems. The variations in the two WN6 systems occur within approximately $-600 \mathrm{~km} / \mathrm{s}$ and $+500 \mathrm{~km} / \mathrm{s}$ of the line center, with the shortest timescale for variability being greater than 2 hours.

\section{HIGH-RESOLUTION IUE VARIABILITY IN V444 CYGNI (WN + O6),}

N. St.-Louis, A.J. Willis, \& L.J. Smith.

The ultraviolet wind eclipse spectrum of V444 Cygni (ratio of the spectra at phases 0.0 and 0.5 ) shows selective absorption in CIV $\lambda 1549.0$, He II $\lambda 1640.4$, and N IV $\lambda 1718.55$ and numerous absorption features forming a large depression at $\lambda \lambda<1500 \AA$ Using experimental wavelengths and intensities of $\mathrm{Fe} \mathrm{V}$ and $\mathrm{Fe}$ VI transitions with each line broadened to a gaussian profile (FWHM $=1000 \mathrm{~km} / \mathrm{s}$ ) and added linearly, we show that the $\lambda \lambda<1500 \AA$ absorption is largely due to Fe V, Fe VI. However, to achieve a good fit required shifting the resulting spectrum by $-1100 \mathrm{~km} / \mathrm{s}$.

\section{SEARCH FOR ULTRAVIOLET VARIABILITY IN HD 151932 (WR 78),}

N. St.-Louis, L.J. Smith, \& A.J. Willis.

A series of 16 IUE spectra of HD 151932 obtained over 4 consecutive days in May 1988 shows no significant variability, but the SiIV $\lambda \lambda 1393,1402$ P Cygni profiles in these spectra show substantial differences compared to an archival spectrum secured in 1980. The $1988 \mathrm{Si} I V$ lines show an excess absorption compared to 1980 , extending a further $\sim 350 \mathrm{~km} / \mathrm{s}$. These differences are similar in character to the Si IV profile variations previously reported for HD 96548.

\section{INTENSIVE PHOTOMETRY OF SOUTHERN WOLF-RAYET STARS,}

\section{L.A. Balona \& J. Egan..}

Intensive photometry of $17 \mathrm{~W}-\mathrm{R}$ stars shows no evidence for $\mathrm{p}$-mode pulsations. In WR 6 and WR 40, two simultaneous periods consistent with g-mode pulsations are found. Many W-R stars show single-period coherent light variations, but in some cases it is unlikely that these can be attributed to binary effects.

\section{NEW RESULTS ON THE PHOTOMETRIC VARIABILITY OF TWO WN8 STARS,} E. Gosset, J.-M. Vreux, J. Manfroid, C. Sterken, \& M. Remy.

We present recent results on the variability of WR 40 and WR 16 . For WR 40 , a detailed analysis is in press (M.N.R.A.S.). For WR 16, we present the first extensive, reliable data set and show that the variability of WR 16 is not as simple as usually thought. The power spectrum is dominated by a peak corresponding to a period of about 10 days, but other significant peaks cannot be neglected. We suggest that the two periodicities discovered by Manfroid et al. (1987, Astron. Astrophys. 185, L17) in their differential photometry could be attributed to the comparison star HD 86000 . 
RECURRENT DUST FORMATION BY WOLF-RAYET STARS,

P.M. Williams, K.A. van der Hucht, \& P.S. Thé.

We present new data in our continuing study of Wolf-Rayet stars which show variations in their infrared fluxes attributable to variations in the rates of dust formation in their winds. There are four established examples:

WR $140=$ HD 193793 (WC7 + O4-5): variation periodic with orbit $(2900 \mathrm{~d})$

WR $137=$ HD 192641 (WC7 + O): last maximum 1984.5, interval $\sim 12$ y

WR 48a (WC8): last maximum 1979, interval > 9 y

WR $70=$ HD 137603 (WC8 + B0): perhaps periodic $(\sim 400 \mathrm{~d}$ ?)

The variation of WR 140 is well linked to its orbital motion, the infrared outbursts occurring near the time of periastron passage; WR 70 and WR 137 are also binaries. There are other candidates whose variation is still being established.

\section{DISPLACED He I ABSORPTION LINES IN WOLF-RAYET STARS: REVISIONS TO $\mathrm{V}_{\infty}, P . M$. Williams \& P.R.J. Eenens.}

We present new spectra of six WC 7--9 stars showing the $2.058 \mu \mathrm{m} \mathrm{He} \mathrm{I} 2 \mathrm{~s}-2 \mathrm{p}$ absorption line displaced by velocities around 0.7 of the stars' terminal velocities derived conventionally. It is argued that these lines are formed in the outermost regions of the winds and give more accurate representations of the wind terminal velocities than the P Cygni profiles of saturated CIV lines observed in the UV. Consequently, mass-loss rates derived for these stars must be reduced in proportion.

HD 5980: A WR + WR BINARY,

V.S. Niemela.

The radial velocities of emission and absorption lines in the spectrum of HD 5980, the brightest W-R star in the SMC, imply that this eclipsing binary has two WN-type components, and probably a line-of-sight O-type neighbor. Both components of the WN4.5+ WN3 binary are more massive than $40 M_{\odot}$.

HUNTING FOR FAINT NEW GALACTIC WOLF-RAYET STARS, M.M. Shara, M. Potter, A.F.J. Moffat, \& L.F. Smith.

Broadband-narrowband He II $\lambda 4686$ UK Schmidt direct plate pairs of a nine-squaredegree region in Carina have been digitized and searched for faint new Wolf-Rayet stars. A ctalog of one million stars located on each plate yielded one hundred candidates. Eight new W-R stars were spectroscopically confirmed: six are WN and two are WC.

\section{WOLF-RAYET STARS AND THE LUMINOUS STAR CONTENT OF GIANT HII REGIONS IN M 33, L. Drissen, A.F.J. Moffat, \& M.M Shara.}

CCD images of the two largest giant HII regions in $M 33$, obtained with a set of narrowband filters sensitive to strong Wolf-Rayet emission lines, reveal eight $\mathrm{W}-\mathrm{R}$ candidates in NGC 604 and nine in NGC 595. The flux in their emission lines is similar to that of normal Galactic W-R stars. The WR/O number ratio in NGC $604(\approx 0.1)$ is similar to the general Galactic ratio, but seems higher in NGC 595. 